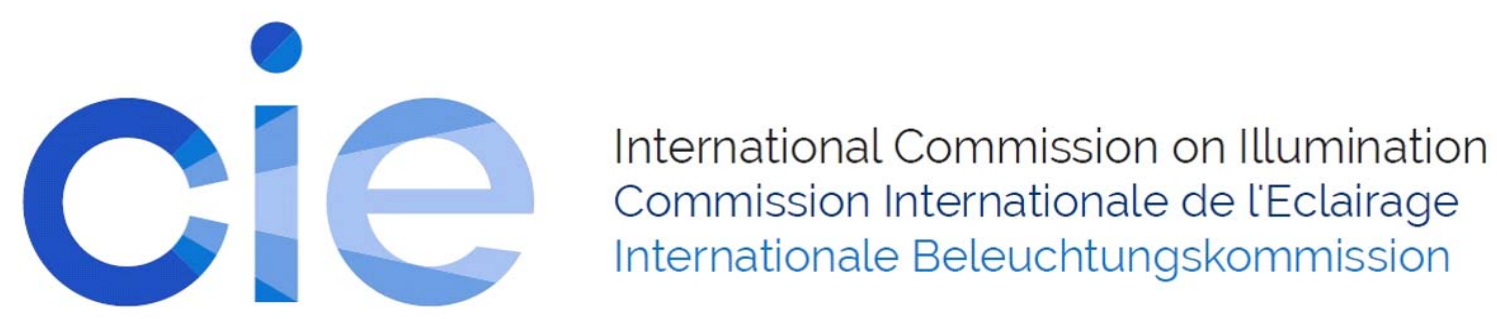

PO044

\title{
APPLICATION OF CIE 13.3-1995 WITH ASSOCIATED CRI- BASED COLOUR RENDITION PROPERTIES
}

\author{
C Teunissen et al.
}

DOI 10.25039/x46.2019.PO044

from

CIE x046:2019

Proceedings

of the

29th CIE SESSION

Washington D.C., USA, June 14 - 22, 2019

(DOI 10.25039/x46.2019)

The paper has been presented at the 29th CIE Session, Washington D.C., USA, June 14-22, 2019. It has not been peer-reviewed by CIE.

(C) CIE 2019

All rights reserved. Unless otherwise specified, no part of this publication may be reproduced or utilized in any form or by any means, electronic or mechanical, including photocopying and microfilm, without permission in writing from CIE Central Bureau at the address below. Any mention of organizations or products does not imply endorsement by the CIE.

This paper is made available open access for individual use. However, in all other cases all rights are reserved unless explicit permission is sought from and given by the CIE.

CIE Central Bureau

Babenbergerstrasse 9

A-1010 Vienna

Austria

Tel.: +4317143187

e-mail: ciecb@cie.co.at

www.cie.co.at 


\title{
APPLICATION OF CIE 13.3-1995 WITH ASSOCIATED CRI-BASED COLOUR RENDITION PROPERTIES
}

\author{
Teunissen, C. ${ }^{1}$, Denneman, J. ${ }^{2}$, Mukai, K. ${ }^{3}$, Wang, S. ${ }^{4}$ \\ ${ }^{1}$ Signify, Eindhoven, NETHERLANDS, ${ }^{2}$ Good Light Group, Eindhoven, NETHERLANDS, \\ ${ }^{3}$ Panasonic, Osaka, JAPAN, ${ }^{4}$ China Academy of Building Research, Beijing, CHINA
}

kees.teunissen@signify.com

DOI 10.25039/x46.2019.PO044

\begin{abstract}
Based on the work completed within CIE Division Reporter 1-68, the Global Lighting Association published a document and an associated calculation tool, that describes the precise procedure for computing the colour rendering indices, $R_{\mathrm{a}}$ and $R_{i}$. In addition, the document includes a colour gamut index $\left(G_{a}\right)$ to indicate if the colour gamut of the test source becomes smaller or larger than the colour gamut of the reference illuminant, chroma indices $\left(C_{i}\right)$ to indicate if the chroma of the test-colour samples will decrease or increase (leading to a less or more colourful appearance of object colours), and $\Delta h_{i}$ to indicate changes in hue-angle. Together with $R_{\mathrm{a}}$, the described properties may be useful to objectively explain why object colours can appear differently when illuminated under light sources with similar CCT and $R_{\mathrm{a}}$ values. This paper provides an overview of the CRI-based colour rendition properties and the importance of the accompanying calculation tool.
\end{abstract}

Keywords: Colour Rendering, Colour Rendition, Colour Gamut, Colour Shifts, Chroma Changes, Correlated Colour Temperature, Wavelength Range, Sampling Interval

\section{Introduction}

The CIE general colour rendering index $\left(R_{\mathrm{a}}\right)$, as defined in CIE 13.3-1995 (CIE, 1995), is widely adopted and used by the lighting industry, in product specification sheets, in regulatory documents and in international and regional standards and specifications. $R_{\mathrm{a}}$ represents an average shift in colour appearance for a set of eight moderately saturated test-colour samples (TCS) under a test light source in comparison to a reference illuminant, having the same correlated colour temperature (CCT) as the test light source. Neither $R_{\mathrm{a}}$ nor the special colour rendering indices, $R_{i}$, provide information on the direction of the colour shifts. However, it is well-known that the direction of the colour shifts (e.g., resulting in an increase or decrease of the colourfulness) can also contribute to the appreciation of perceived object colours. In addition, some people incorrectly assume that light sources with high $R_{\mathrm{a}}$ provide superior colour quality ("higher is better") and that two light sources with similar CCT and $R_{\mathrm{a}}$ values should have equal colour rendition performance. At this moment, there is no international (CIE) standard that provides additional information, beyond CCT \& CRI, with which differences, in colour rendition, between light sources (with similar CCT and $R_{\mathrm{a}}$ values) can be objectively characterised. Without these additional, internationally standardized, objective measures, it will remain difficult, for non-experts in the field of colour science (e.g. normal consumers), to compare the colour rendition performance between white-light sources in all aspects.

In 2017, the CIE published Technical Report 224 "CIE 2017 Colour Fidelity Index for accurate scientific use" (CIE, 2017), describing a scientifically more accurate fidelity index, $R_{\mathrm{f}}$, prepared by CIE Division 1 Technical Committee (TC) 1-90. As mentioned in the report, practical applicability of $R_{\mathrm{f}}$ is still under evaluation and the development of a harmonized set of colour quality measures for assessing perception-related effects beyond fidelity has been proposed. The new CIE 2017 colour fidelity index, $R_{\mathrm{f}}$, is not recommended as a replacement of the CIE general colour rendering index, $R_{\mathrm{a}}$, neither for the purpose of rating and specification of products nor for regulatory or other minimum performance requirements. Consequently, $R_{\mathrm{a}}$ will continue to be used as the global measure for colour fidelity until the CIE completes this work. 
Meanwhile, the lighting associations, such as the Global Lighting Association (GLA, 2015), LightingEurope (LightingEurope, 2014), and the Middle East Lighting Association (MELA, 2016), expressed in their respective position statements the urgent need for additional colourrelated specification items that can supplement the well-established $R_{\mathrm{a}}$.

Based on the work completed within CIE Division 1 Reporter DR 1-68 "A Gamut Area Measure and Colour-shift Graphic, based on CIE 13.3-1995", the GLA published a document (GLA, 2018a) and an associated calculation tool (GLA, 2018b), that describes the procedure for computing the CRI values, $R_{\mathrm{a}}$ and $R_{i}$, and, in addition, it provides information on the direction of the colour shifts for all CIE 13.3-1995 test-colour samples (GLA, 2019). The Excel calculation tool is provided for the users of CRI, because the old DOS-based tool, provided by CIE in the past, may no longer run on modern computers. Furthermore, the calculation tool ensures a consistent calculation of the correlated colour temperature (CCT) and all CRI values. CIE publications 13.3-1995 (CIE, 1995) and 224:2017 (CIE, 2017) do not specify the exact procedure for calculating the CCT, whilst the CCT is used to generate the reference illuminant (a blackbody radiator below $5000 \mathrm{~K}$ and a phase of daylight at $5000 \mathrm{~K}$ and above). Small variations in CCT, e.g. due to differences in wavelength range or wavelength interval, may impact the CRI values, which became apparent during the update of CIE publication 15 "Colorimetry" (CIE, 2004) in TC 1-85. The CCT and CRI values for the CIE illuminants, between participating TC 1-85 members, were slightly different, initiating the discussion which values are the "correct" ones to be included in the technical report. The latest version of CIE publication 15 (CIE, 2018) therefore indicates that "an agreement about details of the computing method is necessary to avoid undesirable discrepancies between computed correlated colour temperatures". Providing an easy to use reference calculation tool is therefore important to enable a consistent reporting of the CCT, CRI, and additional index values.

The associated CRI-based colour rendition properties, described in the GLA publication (GLA 2018a), provide information on the direction of the colour shifts. To retain the characteristics of $\mathrm{CRI}$, the associated colour rendition properties are based on the same CIE 1964 uniform scale coordinates $\left(U^{*}, V^{*}\right)$ as for calculating the CRI values (CIE, 1995). The described properties include a colour gamut index $\left(G_{a}\right)$ to indicate if the colour gamut of the test source becomes smaller or larger than the colour gamut of the reference illuminant (this is an indication if the eight test-colour samples will appear, on average, less or more saturated for the test source in comparison to the reference illuminant), it includes chroma indices $\left(C_{i}\right)$ to indicate if the chroma (related to colour saturation) of the individual test-colour samples will be reduced or enhanced (leading to a less or more colourful appearance of object colours), and it includes hue-angle changes $\left(\Delta h_{i}\right)$ for all individual test-colour samples. Together with $R_{\mathrm{a}}$, the associated colour rendition properties may be useful to objectively explain why object colours can still appear differently when illuminated under light sources with similar CCT and $R_{\mathrm{a}}$ values.

It should be noted that in addition to the method described in the GLA publication (GLA, 2018a), other methods have been proposed to augment colour fidelity with gamut area, chroma and/or hue metrics, including IES TM-30 (IES, 2018), but none of these methods is compatible with $\mathrm{CRI}$ (as requested by the lighting associations). Furthermore, as also indicated in the disclaimer of the GLA publication (GLA, 2018a), the GLA does not prescribe mandatory use of any method, but the GLA document with the CRI-based colour rendition properties is provided for evaluation and voluntary usage by members of the lighting industry and other interested parties and can serve as input for a future global colour rendition standard. As mentioned before, the calculation tool (GLA, 2018b) is provided as a reference and can be used for an easy and consistent calculation of the CCT, distance to the blackbody locus $\left(D_{\mathrm{uv}}\right)$ and all CRI-based colour rendition values.

This paper presents the most important details of the CRI-based colour rendition properties, described in the GLA publication (GLA, 2018a), and the importance of the accompanying calculation tool (GLA, 2018b).

\section{Computation Framework}

The indices described in the GLA publication are all based on Clause 5 of CIE technical report 13.3 (CIE, 1995). For the GLA calculation tool (GLA, 2018b) some specific implementation 
choices have been made, which were not detailed in CIE 13.3. These choices are described in the following subsections $(2.1-2.3)$.

\subsection{Input wavelength range and spectral sampling interval}

In the GLA calculation tool (GLA, 2018b), as per CIE recommendation (CIE, 2004), to avoid interpolation artefacts for peaked spectra (spectra with narrow band emission peaks), the input spectral power distribution (SPD) is not changed, but instead all test-colour samples' (TCS) spectral radiance factors (SRF) are matched to the wavelength range and sampling interval of the input SPD. Furthermore, the CIE 1931, 2-degree, colour-matching functions (CMFs) published in Table 1 of ISO 11664-1:2007(E)/CIE S 014-1/E:2006 (ISO/CIE, 2007) are used in the calculation tool. The values for the CIE 1931 standard colorimetric observer are given at 1 $\mathrm{nm}$ wavelength intervals from $360 \mathrm{~nm}$ to $830 \mathrm{~nm}$ with 7 significant digits. Linear interpolation is used in the calculation tool when values at intermediate (non-integer) wavelength intervals are required.

All 14 test-colour samples described in clause 5.4 (and clause 8 , Tables 1 \& 2) in CIE 13.3 (CIE, 1995) are included in the calculation tool, covering the whole visual wavelength range $(360 \mathrm{~nm}$ to $830 \mathrm{~nm}$ ). Because the TCS are tabulated with $5 \mathrm{~nm}$ increments, linear interpolation was used to store the spectral radiance factors with $1 \mathrm{~nm}$ intervals.

NOTE One optional test-colour sample (TCS 15), East Asian skin (JIS, 1990), is included in the GLA calculation tool. If desired, this TCS can be replaced by another user defined TCS with spectral radiance factors covering the whole visual wavelength range $(360 \mathrm{~nm}-830 \mathrm{~nm})$ and with $1 \mathrm{~nm}$ increments.

\subsubsection{Importance of wavelength range}

To determine the importance of the wavelength range of the input SPD, calculations were done with 220 arbitrarily selected SPDs with spectral data available between $380 \mathrm{~nm}$ and $780 \mathrm{~nm}$ with $1 \mathrm{~nm}$ increments (see Figure 2 for an overview of the SPDs used for the analysis).

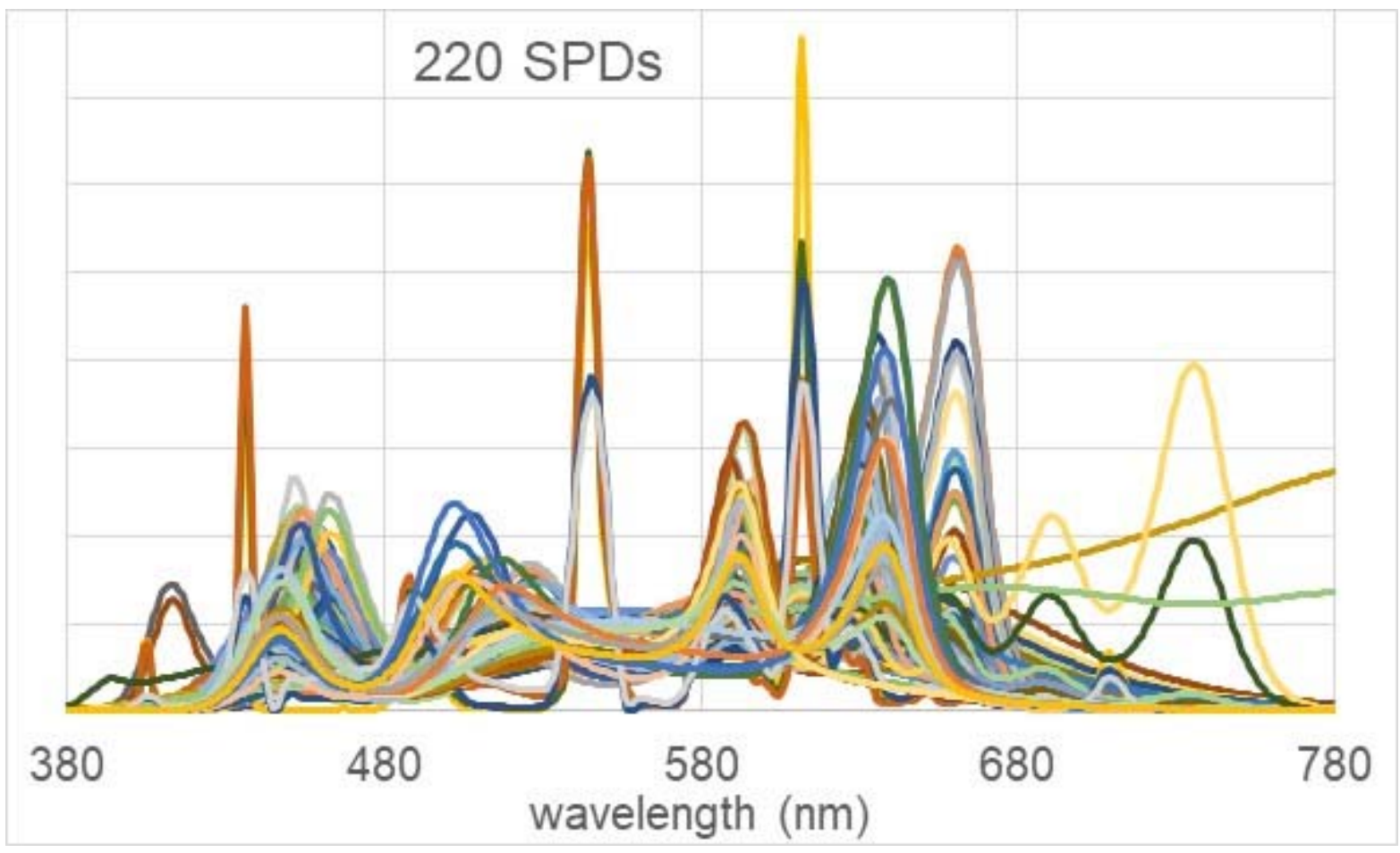

Figure $1-220$ arbitrarily selected SPDs with data in the wavelength range $380 \mathrm{~nm}-780 \mathrm{~nm}$, sampled with $1 \mathrm{~nm}$ intervals. 
The CCT and $R_{\mathrm{a}}$ values for the 220 SPDs were calculated for the full range (380 nm $-780 \mathrm{~nm}$ ) and for a reduced wavelength range $(400 \mathrm{~nm}-700 \mathrm{~nm})$. The latter range was selected because spectral radiance factors are often only measured in this wavelength range. As can be observed in Figure 2, the difference in calculated CCT can be up to $10 \mathrm{~K}$, whereas the difference in $R_{\mathrm{a}}$ values can be up to 0,2 . For product specification purposes, this is accurate enough, but for verification purposes (e.g. values in CIE technical reports) these differences are not acceptable.
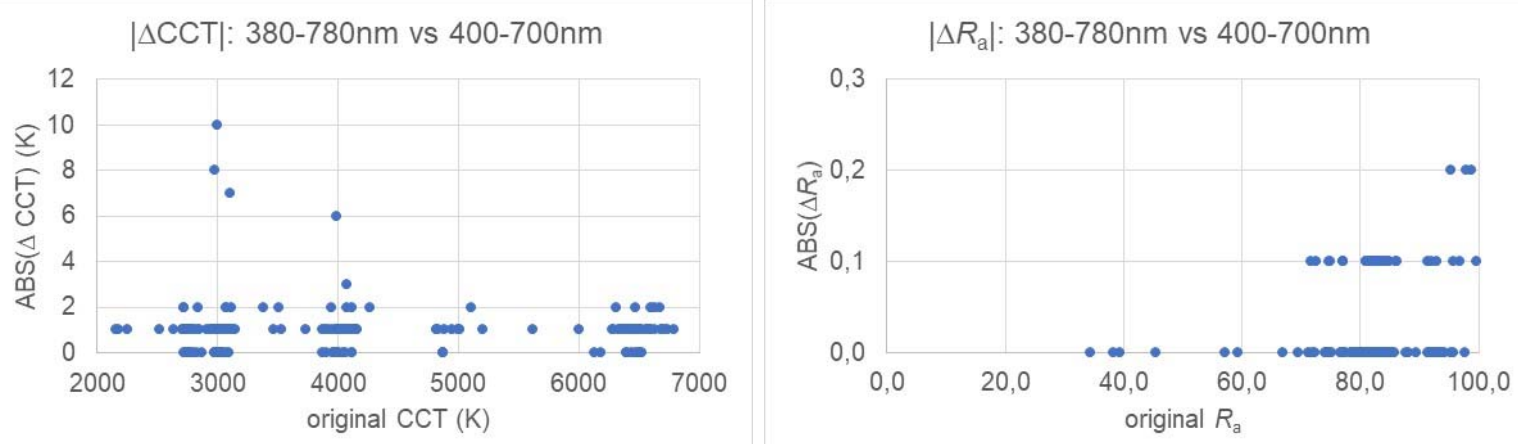

Figure 2 - Absolute differences in CCT and $R_{\mathrm{a}}$ values for 220 SPDs when calculated for the wavelength ranges $380 \mathrm{~nm}-780 \mathrm{~nm}$ (original) and $400 \mathrm{~nm}-700 \mathrm{~nm}$.

The GLA calculation tool is designed for the input spectral range from $360 \mathrm{~nm}$ to $830 \mathrm{~nm}$. The input spectral power distribution (SPD) of the test light source does not have to fill this entire range, but it should have a sufficiently large wavelength range covering at least $380 \mathrm{~nm}$ to 780 $\mathrm{nm}$, conforming to the recommended practice in CIE publication 15 (CIE, 2004; CIE, 2018).

\subsubsection{Importance of wavelength sampling interval}

To determine the importance of the wavelength sampling intervals of the input SPD, calculations were also done with the same 220 SPDs. The CCT and $R_{\mathrm{a}}$ values were calculated with SPD data sampled with 1-nm, 5-nm and 10-nm intervals. As can be observed in Figure 3, the difference in calculated CCT can be more than $200 \mathrm{~K}$ (for $10 \mathrm{~nm}$ sampling intervals), whereas the difference in $R_{\mathrm{a}}$ values can be up to 2,2 (again for $10 \mathrm{~nm}$ sampling intervals). For product specification purposes, this is probably also accurate enough (most differences in $R_{\mathrm{a}}$ are less than 0,5 and most differences in CCT less than $50 \mathrm{~K}$ ), but for verification purposes (e.g. values in CIE technical reports) these differences are not acceptable. Obviously, for 3 or 4 primarybased narrow band light sources (LED, laser, or quantum dot), the differences in $R_{\mathrm{a}}$ and CCT values can be even larger.
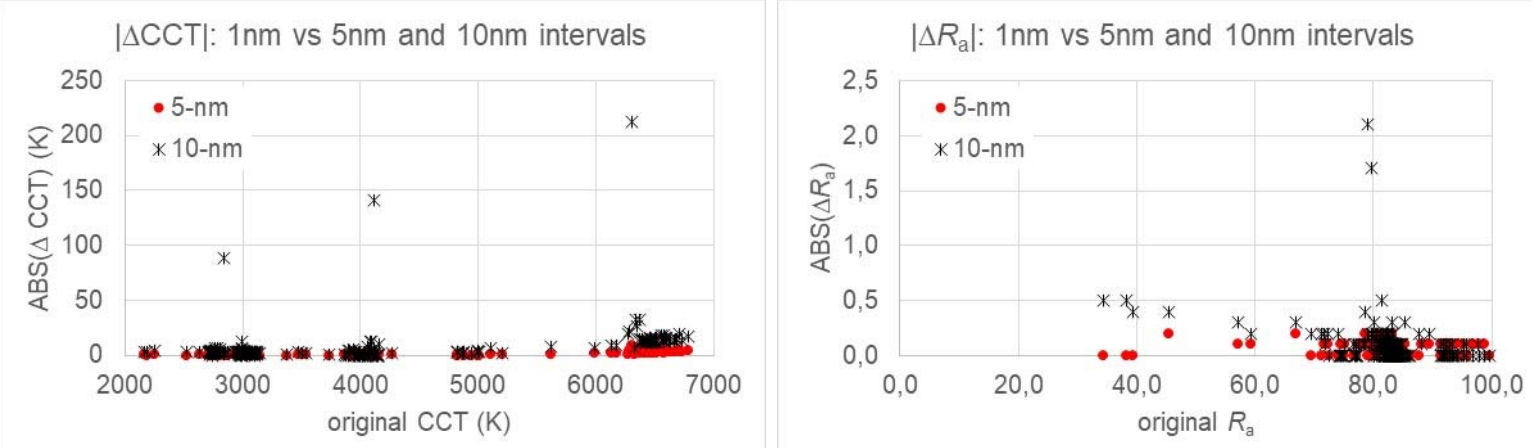

\section{Figure 3 - Absolute differences in CCT and $R_{\mathrm{a}}$ values for 220 SPDs when calculated for the} wavelength range $380-780 \mathrm{~nm}$ and $1 \mathrm{~nm}$ (original) or $5 \mathrm{~nm}$ and $10 \mathrm{~nm}$ sampling intervals.

The input spectral power distribution (SPD) of the test light source should have a sufficiently fine spectral sampling interval (between $1 \mathrm{~nm}$ and $5 \mathrm{~nm}$ ) recommended for accurate colour calculations (CIE, 2004; CIE, 2018). Consequently, the GLA calculation tool accepts all sampling intervals between $1 \mathrm{~nm}$ and $5 \mathrm{~nm}$ (default is $1 \mathrm{~nm}$ ). 


\subsection{Correlated Colour Temperature}

The correlated colour temperature (CCT) of the test white-light source is determined according to clause 9.5 in CIE 15:2004 (CIE, 2004) and clause 9.4 in CIE 15:2018 (CIE, 2018). To obtain accurate and consistent values for the CCT, the combined triangular-parabolic method with a $0,25 \%$ CT-step table, described by Ohno (Ohno, 2014), has been implemented in the GLA calculation tool. This method is accurate to within $1 \mathrm{~K}$ for a CCT range of $1000 \mathrm{~K}$ to $20000 \mathrm{~K}$ and within a distance to the Planckian locus $\left(D_{\mathrm{uv}}\right)$ from $\pm 0,03$ (Ohno, 2014).

NOTE Although the concept of CCT is defined for $\left|D_{\mathrm{uv}}\right|<0,05$ and the $D_{\mathrm{uv}}$ calculation in the GLA tool is accurate to within $1 \mathrm{~K}$ up to $\left|D_{\mathrm{uv}}\right|<0,03$, a smaller range (such as $-0,020 \leq D_{\mathrm{uv}} \leq 0,015$ ) is typically used as a practical limit for white-light sources for general lighting purposes. Another practical limitation in $D_{\mathrm{uv}}$ is imposed by the CRI calculations which become less accurate when $\left|D_{\text {uv }}\right|>$ 0,01 (Teunissen, 2016).

\subsubsection{Importance of the number of entries in the CT-step table}

In the GLA calculation tool, the 0,25\% CT-step table has been implemented which has 1201 entries for the CCT range between $1000 \mathrm{~K}$ and $20000 \mathrm{~K}$. The number of entries can be reduced when the CT-step size is increased: to 303 for a $1 \%$ CT-step table and to 63 for a $5 \%$ CT-step table. Obviously, also the accuracy with which the CCT values for the input SPD are calculated, and consequently the $R_{\mathrm{a}}$ values, will be reduced (see Figure 4 ). When the $1 \%$ CT-step table is used, the differences are quite small, but when the 5\% CT-step table is used, the error in CCT can be up to $20 \mathrm{~K}$ (for the limited set of 220 SPDs) and the error in $R_{\mathrm{a}} 0,3$. Again for practical use in product specifications this might not be a problem, but for verification purposes the results (at least for the 5\% CT-step table) are not accurate enough. Therefore, in the GLA calculation tool, the $0,25 \%$ CT-step table has been implemented.
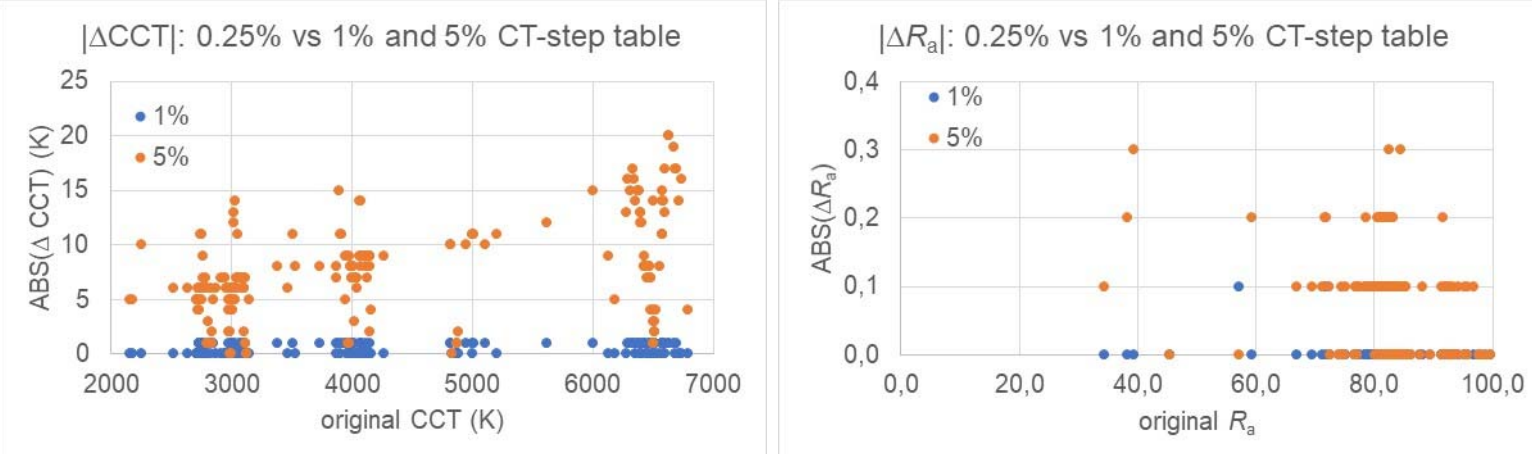

Figure 4 - Absolute differences in CCT and $R_{\text {a }}$ values for 220 SPDs when calculated with $1 \%$ and $5 \%$ CT-step tables (instead of the implemented 0,25\% CT-step table).

\subsection{Reference illuminant}

For calculating the SPD of the reference illuminant, the same procedure is employed as described in clause 5.2 of CIE technical report 13.3 (CIE, 1995). The relative spectral power distribution for the blackbody radiator is calculated according to Appendix E in CIE15:2004 and for daylight according to clause 3.1 (Other illuminants $D$, Item $b$ ) in the same document. For consistency purposes, the reference illuminant in the GLA calculation tool is always calculated for the full wavelength range, $360 \mathrm{~nm}$ to $830 \mathrm{~nm}$, with $1 \mathrm{~nm}$ increments, irrespective of the wavelength range and sampling interval of the input SPD.

\section{Associated CRI-based colour rendition indices}

All associated CRI-based colour rendition indices are derived from the CIE 1964 uniform space coordinates $\left(U^{*}, V^{*}\right)$, which are calculated according to the procedures specified in clauses 5.5 , 5.6, 5.7, and 5.8 in CIE 13.3 (CIE, 1995). Uniform space coordinates $\left(U_{\mathrm{k}, i}^{*}, V_{\mathrm{k}, i}^{*}\right)$ are used for the test source, input SPD, $(\mathrm{k})$ and test-colour sample $(i, i=1 . .15)$ and $\left(U_{\mathrm{r}, i}^{*}, V_{\mathrm{r}, i}^{*}\right)$ for the reference 
illuminant (r). Figure 5 shows an example of the uniform space coordinates for the eight TCS for a test source (input SPD) and the corresponding coordinates for the reference illuminant.

\section{Colour Shift Graphic (CSG)}

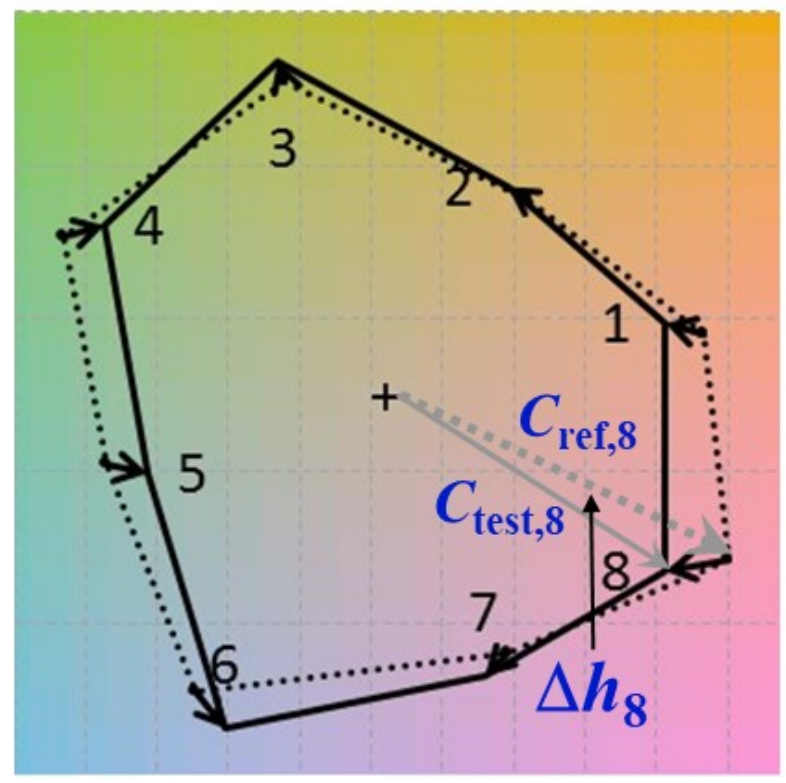

Figure 5 - Example of $U^{*}, V^{*}$ coordinates for the eight TCS for the test source (connected with a solid line) and the reference illuminant (connected with the dotted line) in the CIE 1964 Uniform Colour Space ( $U^{*}$ on the horizontal axis, $V^{*}$ on the vertical axis). The short arrows at the TCS numbers indicate the direction of the colour shifts for the individual TCS. The long arrows indicate the chroma for the test source $\left(C_{\text {test }, 8}\right)$ and for the reference illuminant $\left(C_{\text {ref, }, 8}\right)$ for TCS \#8. The angle between the two arrows $\left(C_{\text {test, }, 8}, C_{\text {ref, }, 8}\right)$ represents the hue-angle change for TCS \#8

$\left(\Delta h_{8}\right)$

\subsection{Colour gamut index}

The colour gamut index, $G_{\mathrm{a}}$, is based on the first eight test-colour samples (same as for $R_{\mathrm{a}}$ ), see Figure 5. It is computed as 100 times the colour gamut for the test source $\left(G_{\mathrm{k}}\right)$ divided by the colour gamut for the reference illuminant $\left(G_{\mathrm{r}}\right)$, see formulae (1).

$$
G_{\mathrm{a}}=100 \times \frac{G_{\mathrm{k}}}{G_{\mathrm{r}}}
$$

\subsection{Chroma indices}

The chroma indices are calculated, for all individual TCS (i), according to formulae (2):

$$
C_{i}=100 \times \frac{C_{\mathrm{k}, i}}{C_{\mathrm{r}, i}}=100 \times \frac{\sqrt{\left(U_{\mathrm{k}, i}^{*}\right)^{2}+\left(V_{\mathrm{k}, i}^{*}\right)^{2}}}{\sqrt{\left(U_{\mathrm{r}, i}^{*}\right)^{2}+\left(V_{\mathrm{r}, i}^{*}\right)^{2}}}
$$

Chroma index $C_{9}$ can be referred to as the red chroma index, because TCS \#9 represents strong red. Similarly, $C_{10}$ represents the yellow chroma index, $C_{11}$ the green chroma index and $C_{12}$ the blue chroma index.

\subsection{Hue-angle changes}

The hue-angle changes are calculated for all individual TCS (i), according to formulae (3): 


$$
\Delta h_{i}=\arctan \left(\frac{V_{\mathrm{r}, i}^{*}}{U_{\mathrm{r}, i}^{*}}\right)-\arctan \left(\frac{V_{\mathrm{k}, i}^{*}}{U_{\mathrm{k}, i}^{*}}\right)
$$

\section{Colour-shift graphic}

The colour-shift graphic (see Figure 6) provides a rough indication for the individual colour shifts for the first eight TCS, represented by the arrows. For visual evaluation purposes, the axes ( $U^{*}$ and $V^{*}$ ) are rescaled by the calculation tool such that the coordinates for the eight TCS just fit within the square area of the $U^{*}-V^{*}$ plane.

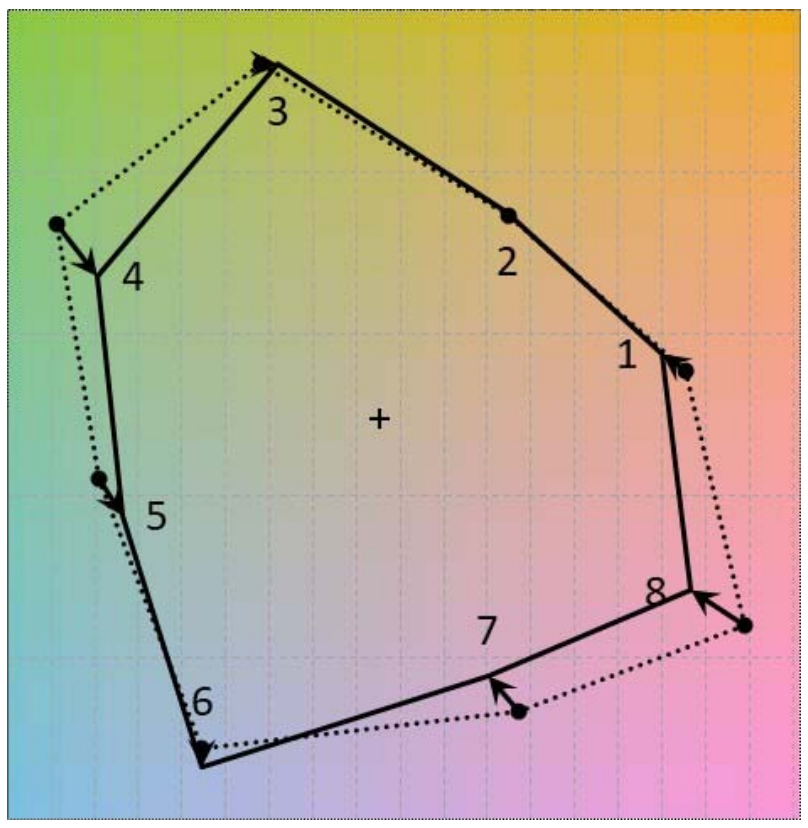

NOTE The numbers in the colour-shift graphic represent the TCS\#, the dotted line connects the coordinates for the reference illuminant and the solid line for the test light source, where the arrows indicate the direction of the colour shifts. The dashed gridlines have the same unit size for the two axes ( $U^{*}$ on the horizontal axis, $V^{*}$ on the vertical axis), which shows possible scale differences for the axes, and the + indicates the origin (coordinates 0,0$)$ of the $U^{*}-V^{*}$ plane.

Figure 6 - Example of colour gamut formed by the eight TCS for a test source (solid line) and the corresponding reference illuminant (dotted line).

\section{Two-dimensional $R_{\mathrm{a}}-G_{\mathrm{a}}$ graphic}

As described in the introduction, $R_{\mathrm{a}}$ represents an average colour fidelity for eight moderately saturated TCS under a test light source in comparison to a reference illuminant, without indicating the direction of the colour shifts. The colour gamut index, $G_{\mathrm{a}}$, provides an indication on whether the colour gamut of the test source is enlarged $\left(G_{\mathrm{a}}>100\right)$ or reduced $\left(G_{\mathrm{a}}<100\right)$ compared to the gamut for the reference illuminant. If colour fidelity is very high $\left(R_{\mathrm{a}}\right.$ close to 100 ), variations in colour gamut are limited (colour shifts must be small to obtain a high $R_{\mathrm{a}}$ value). With increasing colour differences (thus with decreasing $R_{\mathrm{a}}$ values), the possible variation in $G_{\mathrm{a}}$ values increases. This is illustrated in Figure 7 in which $R_{\mathrm{a}}-G_{\mathrm{a}}$ combinations are included, based on slightly more than 100000 SPDs.

The SPDs were created by an 11-channel $(11-\mathrm{CH})$ light source by randomly assigning (8-bit) drive values to the individual channels. Also, $R_{\mathrm{a}}-G_{\mathrm{a}}$ combinations of commercially available fluorescent (FL), high intensity discharge (HID) and light emitting diode (LED) light sources have been included in Figure 7. The following characteristics were used to include the $R_{\mathrm{a}}-G_{\mathrm{a}}$ combinations of the light sources: $1500<T_{\mathrm{cp}}(\mathrm{K})<20000, R_{\mathrm{a}} \geq 60$, and $-0,02 \leq D_{\mathrm{uv}} \leq 0,01$. 


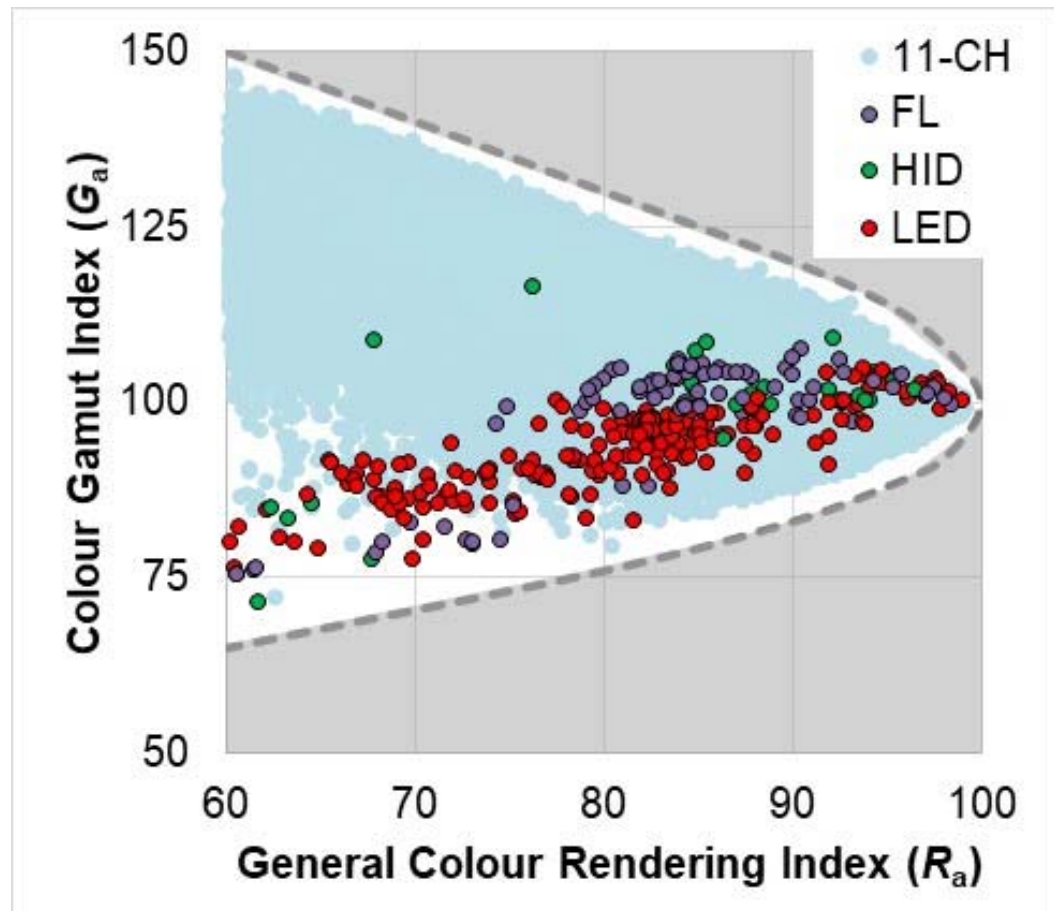

Key:

11-CH: 100000 SPDs, randomly generated with an 11-channel light box

FL: $\quad$ fluorescent type light sources

HID: $\quad$ high intensity discharge type light sources

LED: $\quad$ light-emitting diode type light sources

NOTE The dashed contour line is just an indication and encloses all calculated SPDs. The number of SPDs is limited, so the line anticipates a somewhat broader range of $R_{\mathrm{a}}-G_{\mathrm{a}}$ combinations. It is included to show that the range of possible $G_{\mathrm{a}}$ values increase with decreasing $R_{\mathrm{a}}$ values.

Figure 7 - Illustration of possible $R_{\mathrm{a}}-G_{\mathrm{a}}$ combinations.

\section{Conclusion}

In this paper it has been shown that not only a description of the calculation procedure is important, but also adding some requirements on the input data (wavelength range - at least $380 \mathrm{~nm}$ to $780 \mathrm{~nm}$ - and wavelength sampling interval - default $1 \mathrm{~nm}$ to maximum $5 \mathrm{~nm}$ ), the exact values for the CMF and TCS (without the necessity for interpolation or extrapolation), the exact procedure for calculating the CCT (combined triangular-parabolic method with a $0,25 \%$ CT-step table), and the criteria for calculating the reference illuminant. This helps to enable an accurate reproduction of index values in various laboratories and to compare index values between several calculation tools. This paper also introduced the CRI-based index values that have been published by the Global Lighting Association (GLA, 2018a). As indicated in the GLA document, also other methods have been proposed to augment colour fidelity with gamut area, chroma and/or hue metrics, but these methods are not compatible with CRI. The GLA does not prescribe mandatory use of any method. The described properties are provided for evaluation and voluntary usage by members of the lighting industry and other interested parties. The indices described in the GLA publication can serve as input for a future global colour rendition standard. As requested by the Lighting Associations, the CRI-based indices (for instance $G_{\text {a }}$ and $C_{9}$ ) are fully compatible with $R_{\mathrm{a}}$ and $R_{9}$. Other than the colour rendering indices, the new properties include the direction of the colour shifts and can be used to provide a more complete description of the colour rendition properties of a white-light source. 


\section{References}

CIE 1995. CIE 13.3-1995. Method of Measuring and Specifying Colour Rendering Properties of Light Sources. Vienna, Austria: CIE.

CIE 2004. CIE 15:2004. Colorimetry, $3^{\text {rd }}$ ed. Vienna, Austria: CIE.

CIE 2017. CIE 224:2017. CIE 2017 Colour Fidelity Index for accurate scientific use. Vienna, Austria: CIE.

CIE 2018. CIE 15:2018. Colorimetry, $4^{\text {th }}$ ed. Vienna, Austria: CIE.

IES 2018. IES TM-30-18. IES Method for evaluating light source color rendition. New York (USA): The Illuminating Engineering Society of North America.

ISO/CIE 2007. ISO 11664-1:2007(E)/CIE S 014-1/E:2006. Colorimetry - Part 1: CIE Standard Colorimetric Observers. Vienna, Austria: CIE.

GLA 2015. Global Lighting Association's Position Statement on Colour Rendering Index, 18 September 2015.

GLA 2018a. Global Lighting Association. 2018. Application of CIE 13.3-1995 with Associated CRI-based Colour Rendition Properties. http://www.globallightingassociation.org/library (Accessed 2019).

GLA 2018b. Global Lighting Association. 2018. GLA Calculation Tool for CIE 13.3-1995 CRI and Associated CRI-based Colour Rendition Properties. http://www.globallightingassociation.org/library (Accessed 2019).

GLA 2019. Media Release - Global Lighting Association Publishes Extended Version of Colour Rendering Index, 14 January 2019. http://www.globallightingassociation.org/library (Accessed 2019).

JIS 1990. Japanese Industrial Standard. JIS Z 8726-1990. Method of Specifying Colour Rendering Properties of Light Sources. Translated and Published by Japanese Standards Association, Printed in Japan.

LightingEurope 2014. LightingEurope Position Paper on Color Quality. 6 October 2014.

MELA 2016. The Middle East Lighting Association (MELA) Position Statement on Colour Quality. 4th February 2016.

OHNO, Y. 2014. Practical Use and Calculation of CCT and Duv. LEUKOS, 10(1), 47-55.

TEUNISSEN, C. 2016. Characterizing colourfulness and gamut area of white-light sources in addition to colour fidelity. PROCEEDINGS of the 4th CIE Expert Symposium on Colour and Visual Appearance, 6 - 7 September 2016, Prague, Czech Republic, CIE x043:2016, 236246. 